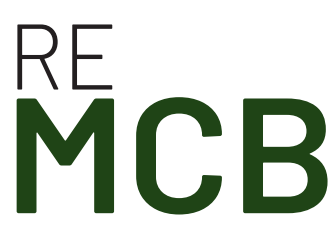

Revista Ecuatoriana de Medicina y Ciencias Biológicas Volumen 42. No. 1, Mayo 2021

\section{Evaluación de la eficacia de cinco desinfectantes comerciales, aplicables en la cadena productiva de musáceas, contra cinco cepas de Fusarium spp.}

\section{Evaluation of the efficacy of five commercial desinfectants, applicable in the production chain of Musaceae, against five strains of Fusarium spp}

Galo Andrés Ordoñez González', Alexander Javier Toaza Mora², Jenifer Marcela Yánez Altuna', Elena Isabel Granda Moreno', Martín Sebastián Marcial-Coba*
1 Pontificia Universidad Católica del Ecuador (PUCE), Quito, Ecuador. ${ }^{2}$ Laboratorio de Fitopatología de la Agencia de Regulación y Control Fito y Zoosanitario - Ecuador (Agrocalidad), Tumbaco, Ecuador.

*Autor de correspondencia: msmarcial@puce.edu.ec

Recibido 30-09-2020

Aceptado 10-02-2021

\section{DOI: 10.26807/remcb.v42i1.884}

e-ISSN 2477-9148

(c) 2021. Este es un artículo publicado en acceso abierto bajo una licencia CC BY 4.0

Como citar este artículo: Ordoñez G, Toaza A, Yánez J, Granda E, Marcial-Coba M. 2021. Evaluación de la eficacia de cinco desinfectantes comerciales, aplicables en la cadena productiva de musáceas, contra cinco cepas de Fusarium spp. Revista Ecuatoriana de Medicina y Ciencias Biológicas 42(1): 11-25. doi: 10.26807/remcb. v42i1.884
Resumen. - El cultivo de banano es uno de los más importantes a nivel mundial y el Ecuador es uno de los países con mayor capacidad de exportación. Esto representa elevados ingresos económicos para el país. Sin embargo, estos cultivos pueden ser afectados nuevamente por el Mal de Panamá, causado por Fusarium oxysporum f. sp. cubense (Foc). Este trabajo está dirigido a determinar la efectividad de desinfectantes frente a cinco cepas distintas de Fusarium spp., con el fin de contribuir a la implementación de procesos de bioseguridad en puntos de ingreso al país y en bananeras. Un total de 5 desinfectantes comerciales fueron seleccionados y su actividad fue evaluada luego de 30 segundos, 1 minuto y 5 minutos de exposición a una suspensión de macroconidios y otra de clamidosporas $\left(10^{6} / \mathrm{mL}\right)$. De los desinfectantes probados, aquel compuesto por glutaraldehído y dos amonios cuaternarios fue el más eficiente al inhibir el desarrollo de Fusarium spp, en la mayoría de las concentraciones evaluadas y en todos los tiempos de exposición. El producto a base de cloruro de alquil dimetil bencil amonio $20 \%$ (amonio cuaternario) y aquel compuesto por ácido hipocloroso 0,05 \% (neuthox) fueron efectivos generalmente en concentraciones mayores o iguales a la recomendada. Ninguno de los desinfectantes de dióxido de cloro fue eficaz inhibiendo el desarrollo de Fusarium spp.

Palabras clave: clamidosporas, concentración mínima inhibitoria, macroconidios, amonio cuaternario, glutaraldehido, ácido hipocloroso, dióxido de cloro.

Abstract. - Banana crop is one of the most important all over the world and Ecuador is one of the countries with the highest export ranges. This represents a significant economic income for the country. However, these crops could be affected by Panama Disease, caused by Fusarium oxysporum f. sp. cubense (Foc). This work aimed to determine the effect of disinfectants solutions against five strains of Fusarium spp., (for the implementation of biosecurity procedures against this disease within farm, ports and airports). A total of 5 commercial disinfectants were screened and their activity was assessed through the exposure of a suspension containing $10^{6}$ macroconidia or chlamydospores $/ \mathrm{mL}$ to different concentrations of the disinfectant agent after 30 seconds, 1 minute and 5 minutes, under laboratory conditions. Among the tested disinfectants, the formulation composed of glutaraldehyde and two different quaternary ammonium compounds was found to be the most effective against Fusarium spp. The product composed of $20 \%$ quaternary ammonium along with the hypochlorous acid-based disinfectant were efficient only when high concentrations were applied, but not when fungal structures were exposed to lower concentrations of the formulation. None of the chlorine dioxide disinfectants was effective, in terms of inhibition of the fungal growth, under the evaluated parameters.

Keywords: chlamydospores, minimal inhibitory concentration, macroconidia, quaternary ammonium, glutaraldehyde, hypochlorous acid, chlorine dioxide. 


\section{Introducción}

El banano es una planta frutal herbácea perteneciente a la familia Musaceae (Al-daour et al. 2019). Las plantaciones de banano se desarrollan de forma óptima en regiones tropicales y subtropicales, con altitudes no mayores a $600 \mathrm{msnm}$ y temperaturas aproximadas de $25{ }^{\circ} \mathrm{C}$, lo que coincide con las condiciones agroclimáticas de regiones como Latinoamérica, Asia (principalmente India y Filipinas), y África occidental (Vásquez 2010; Soto 2011; Gonsabay 2014). Éste es uno de los cultivos de mayor producción a nivel mundial y Ecuador es uno de los países con mayor capacidad de exportación, alcanzando 6685000 toneladas en el año 2019 (FAO 2019). Consecuentemente, esto representa elevados ingresos económicos para el Ecuador como mayor productor mundial de esta fruta.

La existencia de determinados organismos fitopatógenos ha amenazado seriamente la viabilidad del cultivo de banano. Por ejemplo, Fusarium oxysporum f. sp. cubense Raza 1 (Foc R1) es el agente etiológico de la enfermedad denominada "Mal de Panamá" que, durante las décadas del 50 y 60 del siglo anterior, devastó miles de hectáreas de banano de la variedad Gros Michel en el continente americano (Serrano 2012; Rodríguez et al. 2014; Pérez 2015). La sustitución de la variedad Gros Michel por tipos del subgrupo Cavendish, por su resistencia frente a Foc R1, constituyó la solución tomada en aquel entonces por todos los países afectados, incluyendo al Ecuador (Ploetz 2006; Pérez 2015). Asimismo, se ha documentado la existencia de la Raza 2 (Foc R2) que ataca a los bananos de cocción tipo Bluggoe, y la Raza 3 (Foc R3) que ataca a plantas ornamentales del grupo de las heliconias. Además de estas, la Raza 4 (Foc R4) afecta a todos los cultivares de Cavendish, conjuntamente con aquellas variedades sensibles a Foc R1 y Foc R2 (Dita et al. 2018). En función de la región geográfica en las que Foc R4 afecte a la variedad Cavendish, se reconocen dos divisiones del patógeno (Villaverde 2018). F. oxysporum f. sp. cubense Raza 4 división subtropical (Foc R4ST) es el causante de la enfermedad en zonas subtropicales cuando el cultivo se encuentra bajo condiciones de estrés, mientras que F. oxysporum f. sp. cubense Raza 4 división tropical (Foc R4T) es patogénico en condiciones climáticas de regiones tropicales y subtropicales (Dita et al. 2018). Desde la aparición de Foc R4T en el Sudeste Asiático a inicios de los 90's se ha constituido una seria amenaza para la industria bananera (Hwang y Ko 2004; Angel y Rodríguez 2016). En ese contexto, Foc R4T ha afectado seriamente a los cultivares de Cavendish en el Sur de Asia, Asia Pacífico, Sur de Australia, África y recientemente Colombia en América del Sur (ONU 2019), comprometiendo seriamente la supervivencia de los cultivos y consecuentemente conduciendo a grandes pérdidas económicas por parte de los principales exportadores de la fruta (Amani y Avagyan 2014). Ante la confirmación de la presencia de Foc R4T en cultivos de banano en Colombia en el mes de agosto de 2019, se incrementó la preocupación de los países vecinos con respecto a la potencial diseminación del patógeno en la región (ICA 2019; Miranda 2019). Hasta el mes de agosto del 2020 en La Guajira - Colombia, se reportaron aproximadamente 213 hectáreas, actualmente en cuarentena, afectadas por el hongo (ICA 2020).

A diferencia de otros organismos fitopatógenos que requieren de tejidos del hospedador para sobrevivir, las esporas de Fusarium oxysporum pueden ser transportadas, con gran facilidad, por medio de zapatos, equipos, equipajes, herramientas o cualquier utensilio que tenga contacto con el suelo contaminado con las esporas del hongo (Dita et al. 2010). Adicionalmente, las estructuras de este hongo pueden permanecer viables en el suelo por periodos prolongados de tiempo (Rodríguez et al. 2014; Dita y Martínez De La Parte 2014). Por ejemplo, las clamidosporas de Foc R4T sobreviven por más de 30 años, hasta encontrar las condiciones adecuadas para su germinación y desarrollo, lo que puede incluir el contacto con el hospedador (Ángel y Rodríguez 2016; Dita et al. 2013).

Dicho esto, la eventual entrada y diseminación de este patógeno al Ecuador tendría consecuencias devastadoras tanto económicas como en términos de seguridad alimentaria (Molina et al. 2009; Ángel y Rodríguez 2016). Una de las medidas más rápidas y simples para controlar la diseminación de Foc, es el uso de desinfectantes en implementos, equipos, zapatos, y otros objetos que puedan tener contacto con esporas fúngicas (Pérez y De la Parte 2015; Cedeño 2020). Estas formulaciones químicas se emplean con el propósito de inhibir el desarrollo de microorganismos sobre una superficie inerte u objeto inanimado, al ejercer su acción en distintas 
estructuras de las células microbianas, limitando así la contaminación cruzada en múltiples cadenas de producción (Loor y Gordon 2013). La eficiencia de estos productos depende de la composición, la concentración, la temperatura y del tiempo de acción (Burguet et al. 2013). Esta también varía en función del organismo diana. Así por ejemplo, algunos hongos filamentosos son más resistentes a compuestos clorados, comparados con bacterias no esporuladas (Guentzel et al. 2010). La efectividad de un mismo desinfectante puede mostrar diferencias significativas, incluso, frente a distintas especies de hongos, siendo Fusarium spp. uno de los géneros más resistentes (Rood et al. 2018; Guerra et al. 2019). El pH es otro valor para tener en cuenta, ya que las formas ionizadas de los agentes disociables son más efectivas al permear con mayor facilidad a través de las membranas biológicas (los agentes aniónicos tienen mayor actividad a pH ácido y los catiónicos a pH alcalino) (Meldrum et al. 2013).

Previamente se han realizado estudios relacionados a la eficiencia de desinfectantes comerciales frente a diferentes cepas de F. oxysporum en distintas plantaciones. Estas investigaciones han evidenciado resultados variables. Por ejemplo, mientras productos a base de detergentes (ácido alquilbencenosulfónico, dietanolamida de coco, piridina-2-14 tiol 1-óxido y sal de sodio), como Castrol Farmcleanse (Castrol Australia Pty Ltd., Docklands, VIC, Australia), han mostrado eficiencia en el control del crecimiento de Fusarium oxysporum f. sp. vasinfectum en plantaciones de algodón (Moore et al. 2001; Bennett et al. 2011), otros ensayos más recientes han demostrado que no son efectivos previniendo la germinación y el crecimiento de Fusarium oxysporum f. sp. cubense raza 4 subtropical (Nel et al. 2007; Pérez 2015). En contraste, los productos a base de amonio cuaternario presentan mayor eficiencia inhibiendo el desarrollo de varias cepas de Fusarium oxysporum f. sp. cubense raza 1 y raza 4, divisiones subtropical y tropical, en diferentes condiciones de ensayo ( $\mathrm{Nel}$ et al. 2007; Meldrum et al. 2013). Se conoce también que el agua neutral electrolizada (NEW por sus siglas en inglés), cuyo ingrediente activo es ácido hipocloroso, es efectivo reduciendo el número de conidios de Fusarium spp. en semillas y frutas de tomate infectadas artificialmente (Vásquez et al. 2016; Guerra et al. 2019). Asimismo, la eficacia de formulaciones a base de dióxido de cloro frente a conidios y clamidosporas de Fusarium oxysporum también ha sido evaluada, observándose resultados variables (Copes et al. 2004; Scarlett et al. 2016).

En función de la mencionada variabilidad de resultados, se considera necesario evaluar diversos desinfectantes disponibles en el mercado local, con el propósito de seleccionar productos capaces de inhibir el desarrollo de cepas de Fusarium spp. y así obtener resultados orientados a la delimitación de fungicidas que podrían evaluarse y aplicarse como medidas de prevención en plantaciones y puntos de ingreso al país, como puertos y aeropuertos frente al potencial ingreso de Foc R4T al Ecuador.

Considerando la amenaza fitosanitaria que Foc R4T representa para los cultivos de banano en el Ecuador, el principal propósito de esta investigación fue evaluar la eficacia de varios desinfectantes comerciales, en función de la concentración y el tiempo de contacto, frente a un amplio espectro de cepas de Fusarium spp., en conexión con la estrategia de prevención ante la eventual entrada de éste patógeno al país. Este artículo podría constituir una herramienta capaz de aportar información confiable para el establecimiento de medidas preventivas frente a Foc R4T en la cadena productiva de musáceas y en distintos puntos de ingreso al país, como puertos y aeropuertos.

\section{Materiales Y Métodos}

Selección de cepas fúngicas y desinfectantes. - Las cepas seleccionadas para el presente estudio fueron provenientes de las colecciones de cultivos pertenecientes a los laboratorios de Fitopatología de la Agencia de Regulación y Control Fito y Zoosanitario (Agrocalidad) y de la Facultad de Ciencias Exactas y Naturales de la Pontificia Universidad Católica del Ecuador (PUCE). Se utilizaron 5 cepas de Fusarium spp., previamente aisladas y rigurosamente identificadas (Tabla 1).

Se utilizaron 5 desinfectantes comerciales con diferentes composiciones químicas (Tabla 2). De cada uno de los desinfectantes se consideraron seis diferentes concentraciones especificadas 
Tabla 1. Cepas empleadas en el estudio

\begin{tabular}{cccc}
\hline Código & Especie & $\begin{array}{c}\text { Regiones secuenciadas } \\
\text { para identificación del } \\
\text { organismo }\end{array}$ & $\begin{array}{c}\text { Cultivo de } \\
\text { origen }\end{array}$ \\
\hline FP 19-0958 & F. oxysporum & ITS y TEF & Banano \\
B-MB-01 & F. oxysporum & ITS y TEF & Banano \\
FP 15-0317 & F. oxysporum & ITS y TEF & Frutilla \\
FP 14-062 & F. incarnatum - equiseti & ITS y TEF & Soya \\
YC3 & F. oxysporum & ITS y TEF & Uvilla \\
\hline
\end{tabular}

Cepas de Fusarium spp. empleadas en los ensayos de eficacia de desinfectantes. F. oxysporum YC3 provino de la colección de cultivos del laboratorio de Fitopatología de la PUCE, mientras que F. oxysporum FP 19-0958, B-MB-01 y FP 15-0317 junto con F. incarnatum - equiseti FP 14062 pertenecen a la colección de cultivos del laboratorio de Fitopatología de Agrocalidad

en la (Tabla 2).

Suspensión de macroconidios y clamidosporas. - A partir de crioviales conservados a $-80{ }^{\circ} \mathrm{C}$, cada una de las cepas fue propagada, de forma individual, en 5 cajas Petri conteniendo PDA (Potato Dextrosa Agar). Para esto, los cultivos fueron incubados a $25^{\circ} \mathrm{C}$ durante 10 días. La pureza de cada cepa en el medio de cultivo se determinó mediante análisis microscópico. La suspensión de macroconidios de cada cepa se preparó removiendo el micelio de la superficie de los cultivos utilizando un asa micológica. Éste se transfirió a un matraz Erlenmeyer estéril conteniendo $50 \mathrm{~mL}$ de solución fisiológica de $\mathrm{NaCl} 0.85 \%$ con perlas de vidrio de $3 \mathrm{~mm}$ de diámetro. Esta preparación se homogenizó en un agitador rotatorio durante 30 minutos y luego se filtró el contenido a través de almohadillas de algodón estériles para remover las hifas del patógeno. La concentración fue ajustada a $10^{6}$ macroconidios $/ \mathrm{mL}$, lo que fue confirmado mediante recuento en cámara de Neubauer (AOAC 2005; Davicino et al. 2007; Lin et al. 2016). En cuanto a la suspensión de clamidosporas se siguió la metodología descrita por (Nguyen et al. 2019) con algunas modificaciones. Brevemente, las cepas se propagaron en 5 cajas Petri con medio SNA (Spezieller Nahrstoffarmer Agar) bajo las mismas condiciones descritas arriba. A partir de los cultivos en SNA se transfirió el micelio superficial directamente a frascos con $50 \mathrm{~mL}$ de caldo suelo estéril. Estos se agitaron a 90 rpm en un agitador orbital a temperatura ambiente por 10 días. Se realizaron recuentos en cámara de Neubauer, periódicamente, desde el quinto día hasta llegar a la concentración de $10^{6}$ clamidosporas $/ \mathrm{mL}$. Finalmente, este cultivo líquido se filtró haciendo uso de almohadillas de algodón estériles, con el fin de capturar clamidosporas, y posteriormente se filtró el algodón empleado anteriormente, en sentido inverso, para depositar los propágulos de clamidosporas en 50 mL de solución estéril de $\mathrm{NaCl}$ 0,85 \%.

Ensayo de contacto. - Se empleó la metodología 955. 17 de la Association of Official Analytical Chemists (AOAC 2005). Para ello, se prepararon $5 \mathrm{ml}$ de las diluciones de los desinfectantes (Tabla 2). Conjuntamente se utilizó un control negativo de fenol que permite el crecimiento fúngico a una dilución de 1:70 y un control positivo de fenol que inhibe el desarrollo del hongo a una dilución de 1:60. Los tubos con las distintas diluciones de los desinfectantes fueron temperados en baño María a $25^{\circ} \mathrm{C}$. Luego se colocó, de forma individual, 0,5 mL de la solución stock de macroconidios o clamidosporas en los tubos con las distintas concentraciones de desinfectantes. Transcurrido el tiempo de exposición (30 segundos, 1 y 5 minutos) se inocularon $10 \mu \mathrm{L}$ de la suspensión anterior, en $10 \mathrm{~mL}$ de caldo glucosa suplementado con lecitina 0,07 \% y 0,5\% de polisorbato 80, como agentes neutralizantes de la acción fungistática de los desinfectantes (Fernández-Crehuet et al. 2013). Este procedimiento, en cuanto a los controles, se realizó a un tiempo de exposición de 10 minutos. Los tubos con caldo glucosa modificado se incubaron a $25^{\circ} \mathrm{C}$ por 10 días. A partir de ese momento, se observaron los resultados finales mediante la determinación de la concentración mínima inhibitoria (CMI) de cada producto desinfectante, en función de la turbidez del medio de cultivo. 
Tabla 2. Composición de los desinfectantes y concentraciones de cada producto evaluados en el estudio

\begin{tabular}{|c|c|c|c|c|c|c|}
\hline Desinfectante & \multicolumn{5}{|c|}{ Composición } & $\begin{array}{c}\text { Casa } \\
\text { Comercial }\end{array}$ \\
\hline Agri'germ 1510 & \multicolumn{5}{|c|}{ Glutaraldehído $15 \%$} & Imvab \\
\hline & \multirow{2}{*}{\multicolumn{5}{|c|}{$\begin{array}{l}\text { Cloruro de Alquil Dimetil Bencil Amonio } 8 \% \\
\text { Cloruro de Dimetil Didecil Amonio } 2 \%\end{array}$}} & CIA.LTDA \\
\hline & & & & & & \\
\hline Amonio cuaternario & \multicolumn{5}{|c|}{ Cloruro de Alquil Dimetil Bencil Amonio $20 \%$} & Maymo S.A. \\
\hline Neuthox & \multicolumn{5}{|c|}{ Ácido hipocloroso 0,05 \% } & $\begin{array}{c}\text { Danish Clean } \\
\text { Water S.A. }\end{array}$ \\
\hline Clodos Puro & \multicolumn{5}{|c|}{ Dióxido de cloro 0,0075 \% (75ppm) } & STC S.L.U. \\
\hline \multirow[t]{3}{*}{ Biocidox } & \multicolumn{5}{|c|}{ Dióxido de cloro 10 \% (Tabletas $20 \mathrm{~g}$ ) } & Boeckorp S.A. \\
\hline & \multicolumn{6}{|c|}{ Diluciones (\%) } \\
\hline & C1 & $\mathrm{C2}$ & C3 & C4 & C5 & C6 \\
\hline Agri'germ 1510 & 1,6 & 1,2 & 0,8 & 0,4 & 0,2 & 0,1 \\
\hline Amonio cuaternario & 0,2 & 0,15 & 0,1 & 0,05 & 0,025 & 0,0125 \\
\hline Neuthox & 60 & 45 & 30 & 15 & 7,5 & 3,75 \\
\hline Clodos Puro & 0,58 & 0,43 & 0,29 & 0,15 & 0,075 & 0,038 \\
\hline Biocidox & 0,002 & 0,0015 & 0,001 & 0,0005 & 0,00025 & 0,00013 \\
\hline
\end{tabular}

Valores numéricos corresponden a la concentración del producto en las diluciones consideradas para el ensayo: $\mathrm{C} 1$ - doble de la concentración recomendada; $\mathrm{C2}$ - promedio de la concentración recomendada y del doble de esta; C3 - concentración recomendada por el fabricante; C4 - dilución 1:2 de la concentración recomendada; C5 - dilución 1:4 de la concentración recomendada; C6 dilución 1:8 de la concentración recomendada.

Como método de confirmación se empleó el método de siembra de mohos y levaduras de la Norma ISO 21527-2 (2018) con algunas modificaciones. De forma aleatoria entre las réplicas (por cepa y por desinfectante), se seleccionó uno de los tubos con la concentración más baja de aquellos que presentaron inhibición y se inoculó $100 \mu \mathrm{L}$ de su contenido sobre la superficie en agar PDA, esperando crecimiento nulo de microorganismos después de 10 días de incubación a $25^{\circ} \mathrm{C}$. Por otra parte, se seleccionó de forma aleatoria, uno de los tubos que presentaron crecimiento con la concentración más alta del desinfectante y se inocularon 100 $\mu \mathrm{L}$ de su contenido en agar PDA, con el propósito de evidenciar el desarrollo, únicamente, de Fusarium spp., luego del tiempo de incubación (bajo las condiciones previamente descritas), y así descartar la presencia de microorganismos contaminantes que pudieran interferir en los resultados del ensayo.

Análisis estadístico. -Todos los ensayos experimentales fueron ejecutados por duplicado siguiendo la norma de la AOAC (2005). Para determinar el nivel de significación entre los desinfectantes con la inhibición de Fusarium spp. y predecir la relación de las variables, los resultados se analizaron mediante regresión logística binaria conjuntamente para macroconidios y clamidosporas. Los datos se agruparon en dos grupos: variables independientes (tipo de desinfectante, concentraciones de cada uno y el tiempo de exposición) y variables dependientes (inhibición o desarrollo del hongo). Para ello se hizo uso del software IBM Statistical Package for the Social Science - SPSS 22 (IBM Corporation 2015; Li et al. 2015).

\section{Resultados}

La efectividad de los desinfectantes frente a Fusarium spp. fue medida en base a la concentración mínima inhibitoria (CMI), es decir, la dilución más alta capaz de inhibir el desarrollo de macroconidios y clamidosporas dentro de cada tiempo de exposición. A los 30 segundos de exposición, únicamente Agri'germ 1510 inhibió el crecimiento de macroconidios de todas 
las cepas en la dosis recomendada (producto diluido al 0,8\% equivalente a glutaraldehido $0,12 \%$, cloruro de alquil dimetil bencil amonio 0,064 \%, cloruro de dimetil didecil amonio 0,016 \%). Además, la CMI de este producto osciló entre C6 (0,1%) y C3 (concentración recomendada). En el caso de clamidosporas, la CMI de Agri'germ 1510 frente a cada cepa aumentó a la concentración inmediata superior para todas las cepas evaluadas (Tabla 3). Neuthox inhibió el crecimiento de macroconidios de las cepas FP 19-0958 y FP 15-0317 en la concentración recomendada (C3 - producto diluido al 30 \% equivalente a ácido hipocloroso $0.015 \%$ ), y en las cepas restantes en la dilución C2 (producto diluido al $45 \%$ ). Respecto al ensayo con clamidosporas de Fusarium spp., se requirieron las concentraciones C1 (producto diluido al 60 \%) y C2 (producto diluido al $45 \%$ ) para inhibir el desarrollo de las estructuras fúngicas. La CMI del producto Amonio cuaternario frente a macroconidios, en este tiempo de exposición, fue C1 (producto diluido al 0,2 \%) para todas las cepas, con excepción de FP 19-0958 y B-MB-01. En contraste, frente a clamidosporas este desinfectante impidió el crecimiento de todas las cepas en la misma dilución (C1), exceptuando el aislamiento B-MB-01. Los desinfectantes Clodos Puro y Biocidox no inhibieron la germinación de macroconidios y clamidosporas de las cepas de Fusarium spp. evaluadas, en ninguna concentración y tiempo de exposición (Tabla 3).

Transcurrido un minuto de exposición, la CMI de Agri'germ 1510 frente a macroconidios fue inferior en dos cepas, reduciéndose de C4 (producto diluido al 0,4\%) a C5 (producto diluido al 0,2\%). Este desinfectante fue el único en inhibir el crecimiento de clamidosporas, de cada una de las cepas empleadas, dentro de la concentración recomendada al minuto de contacto. Sin

Tabla 3. Concentración mínima inhibitoria de cada desinfectante inhibiendo el crecimiento de conidios y clamidosporas de 5 cepas de Fusarium spp. a los 30 segundos, 1 minuto y 5 minutos de exposición

\begin{tabular}{|c|c|c|c|c|c|c|c|c|c|c|}
\hline \multicolumn{11}{|c|}{ Concentración Mínima Inhibitoria } \\
\hline \multirow[b]{2}{*}{ Cepas } & \multicolumn{5}{|c|}{ Conidios } & \multicolumn{5}{|c|}{ Clamidosporas } \\
\hline & $\begin{array}{c}\text { Agri'germ } \\
1510\end{array}$ & Neuthox & $\begin{array}{c}\text { Amonio } \\
\text { cuaternario }\end{array}$ & $\begin{array}{l}\text { Clodos } \\
\text { Puro }\end{array}$ & Biocidox & $\begin{array}{c}\text { Agri'germ } \\
1510\end{array}$ & Neuthox & $\begin{array}{c}\text { Amonio } \\
\text { cuaternario }\end{array}$ & $\begin{array}{l}\text { Clodos } \\
\text { Puro }\end{array}$ & Biocidox \\
\hline \multicolumn{11}{|l|}{30 segundos } \\
\hline FP19-0958 & C3 & C3 & $\mathrm{NI}$ & $\mathrm{NI}$ & $\mathrm{NI}$ & $\mathrm{C} 2$ & $\mathrm{C} 2$ & $\mathrm{C} 1$ & $\mathrm{NI}$ & $\mathrm{NI}$ \\
\hline B-MB-01 & C6 & $\mathrm{C} 2$ & $\mathrm{NI}$ & $\mathrm{NI}$ & $\mathrm{NI}$ & C5 & $\mathrm{C} 2$ & $\mathrm{NI}$ & $\mathrm{NI}$ & $\mathrm{NI}$ \\
\hline FP15-0317 & $\mathrm{C} 5$ & $\mathrm{C} 3$ & $\mathrm{C} 1$ & $\mathrm{NI}$ & $\mathrm{NI}$ & $\mathrm{C} 4$ & $\mathrm{C} 1$ & $\mathrm{C} 1$ & $\mathrm{NI}$ & $\mathrm{NI}$ \\
\hline FP 14-062 & $\mathrm{C} 4$ & $\mathrm{C} 2$ & $\mathrm{C} 1$ & $\mathrm{NI}$ & $\mathrm{NI}$ & C3 & $\mathrm{C} 1$ & $\mathrm{C} 1$ & $\mathrm{NI}$ & $\mathrm{NI}$ \\
\hline YC3 & $\mathrm{C} 4$ & $\mathrm{C} 2$ & $\mathrm{C} 1$ & $\mathrm{NI}$ & $\mathrm{NI}$ & C3 & $\mathrm{C} 2$ & C1 & $\mathrm{NI}$ & $\mathrm{NI}$ \\
\hline \multicolumn{11}{|l|}{1 minuto } \\
\hline FP19-0958 & $\mathrm{C} 3$ & C3 & C3 & $\mathrm{NI}$ & $\mathrm{NI}$ & $\mathrm{C} 3$ & $\mathrm{C} 2$ & $\mathrm{C} 1$ & $\mathrm{NI}$ & $\mathrm{NI}$ \\
\hline B-MB-01 & C6 & C3 & $\mathrm{C} 1$ & $\mathrm{NI}$ & $\mathrm{NI}$ & C6 & $\mathrm{C} 2$ & $\mathrm{C} 1$ & $\mathrm{NI}$ & $\mathrm{NI}$ \\
\hline FP15-0317 & C5 & C3 & $\mathrm{C} 2$ & $\mathrm{NI}$ & $\mathrm{NI}$ & C5 & C3 & C3 & $\mathrm{NI}$ & $\mathrm{NI}$ \\
\hline FP 14-062 & C5 & C3 & $\mathrm{C} 2$ & $\mathrm{NI}$ & $\mathrm{NI}$ & C5 & $\mathrm{C} 2$ & $\mathrm{C} 1$ & $\mathrm{NI}$ & $\mathrm{NI}$ \\
\hline YC3 & C5 & C4 & $\mathrm{C} 1$ & $\mathrm{NI}$ & $\mathrm{NI}$ & C5 & C3 & C1 & $\mathrm{NI}$ & $\mathrm{NI}$ \\
\hline \multicolumn{11}{|l|}{5 minutos } \\
\hline FP19-0958 & C4 & C4 & C3 & $\mathrm{NI}$ & $\mathrm{NI}$ & $\mathrm{C} 4$ & C3 & $\mathrm{C} 3$ & $\mathrm{NI}$ & $\mathrm{NI}$ \\
\hline B-MB-01 & C6 & C3 & $\mathrm{C} 1$ & $\mathrm{NI}$ & $\mathrm{NI}$ & C6 & C4 & $\mathrm{C} 1$ & $\mathrm{NI}$ & $\mathrm{NI}$ \\
\hline FP15-0317 & C5 & $\mathrm{C} 4$ & $\mathrm{C} 3$ & $\mathrm{NI}$ & $\mathrm{NI}$ & C5 & C3 & $\mathrm{C} 3$ & $\mathrm{NI}$ & $\mathrm{NI}$ \\
\hline FP 14-062 & C5 & C3 & C3 & $\mathrm{NI}$ & $\mathrm{NI}$ & C5 & C3 & C3 & $\mathrm{NI}$ & $\mathrm{NI}$ \\
\hline YC3 & C5 & C4 & C4 & $\mathrm{NI}$ & $\mathrm{NI}$ & C5 & C3 & C3 & $\mathrm{NI}$ & $\mathrm{NI}$ \\
\hline
\end{tabular}

[C1] doble de la concentración recomendada, [C2] promedio de la concentración recomendada y del doble de esta,

[C3] Concentración recomendada por el fabricante, [C4] Dilución 1:2 de la concentración recomendada, [C5]

Dilución 1:4 de la concentración recomendada, [C6] Dilución 1:8 de la concentración recomendada, [NI] No hay inhibición de conidios y/o clamidosporas 
embargo, no existió diferencia con relación al ensayo efectuado con macroconidios durante el mismo tiempo de exposición En el caso de Neuthox, la concentración recomendada (C3 producto diluido al $30 \%$ ) correspondió a la CMI para macroconidios de todas las cepas, menos para la cepa YC3, cuya CMI correspondió a la dilución C4 (producto diluido al 15 \%). La CMI de este producto para clamidosporas osciló entre C2 (producto diluido al $45 \%$ ) y C3 (producto diluido al $30 \%$ ), siendo mayor en todos los casos a la concentración requerida para inhibir macroconidios. El producto Amonio cuaternario tuvo un efecto inhibitorio sobre el crecimiento de macroconidios y clamidosporas de todas las cepas en las tres primeras concentraciones. No obstante, la concentración más elevada (C1 equivalente a una dilución del 0,2% del producto) fue necesaria para evitar la propagación de clamidosporas, exceptuando la cepa FP 15-0317 cuya CMI (C3 - 30 \%) fue menor en comparación con C2 (producto diluido al 45 \%) que inhibió los macroconidios de esta cepa (Tabla 3).

Al cabo de 5 minutos de exposición, Agri'germ 1510 mostró un efecto fungistático, sobre las diferentes cepas evaluadas, en las diluciones C4 (0,4\%), C5 (0,2 \%) y C6 (0,1\%). La CMI de Agri'germ 1510 frente a macroconidios y clamidosporas no varió con relación al minuto de exposición, con excepción de la cepa FP19-0958, para la cual la CMI disminuyó de C3 (producto diluido al 0,8\%) a C4 (producto diluido al 0,4 \%). La CMI de Neuthox ante la presencia de macroconidios fue inferior para dos cepas (FP19-0958 y FP15-0317), pasando de C3 (30\%), luego de 1 minuto de exposición, a C4 (15\%) tras 5 minutos de exposición. Con respecto al ensayo ejecutado con clamidosporas expuestas a Neuthox, la CMI también decreció con respecto al anterior tiempo de exposición. Vale resaltar el caso de la cepa B-MB-01, para la cual se evidenció que la CMI frente a clamidosporas fue menor que aquella registrada en macroconidios después de exponerse por 5 minutos al desinfectante Neuthox. El producto Amonio cuaternario inhibió a los macroconidios del hongo desde la dilución C4 (0,05\%) hasta C1 (0,2\%), observándose una disminución en la CMI para dos cepas (FP 19-0958 y FP 15-0317). Asimismo, la CMI frente a clamidosporas luego de 5 minutos de exposición al producto amonio cuaternario fue C3 (producto diluido al 0,1\% equivalente a cloruro de alquil dimetil bencil amonio $0.02 \%$ ), excepto la cepa B-MB-01. Igualmente, es necesario mencionar que la CMI frente a ambas estructuras fúngicas no varía dentro de los 5 minutos, excluyendo a la cepa YC3 (Tabla 3).

En base al análisis estadístico realizado en conjunto para conidios y clamidosporas, queda demostrado que el desinfectante con la probabilidad más alta de inhibir el crecimiento de Fusarium spp. es Agri'germ 1510 (Exp-B $=0,12: P=0,000$ ). Asimismo, el aumento del tiempo de exposición está relacionado directamente con una mayor efectividad del desinfectante (Exp-B $=2,650: P=0,000$ ). Finalmente, se demostró que el factor de dilución de los productos se relaciona de forma inversa con su efecto desinfectante (Exp-B $=0,250$ : $P=0,000$ ). Las variables independientes (tipo de desinfectante, concentración y tiempo de exposición) se relacionaron directamente con las variables dependientes (desarrollo o inhibición del hongo). Esto se justificó con el análisis de regresión logística, mostrando que existe un 88,1 \% de probabilidad de acierto en los resultados obtenidos de las variables dependientes (Tabla 4).

A partir de este resultado, se tomó en cuenta la significancia del modelo $(<0,05)$ y el valor de la ecuación de la regresión (Exp-B): si este es $>1$, quiere decir que al aumentar el número de la variable independiente la inhibición del microorganismo se vuelve más probable; si el valor es $<1$, indica que al aumentar el número de la variable independiente la inhibición del microorganismo es menos probable (Tabla 4).

\section{Discusión}

En la evaluación de la eficacia de distintos desinfectantes comerciales en relación al desarrollo de propágulos de varias cepas de Fusarium spp. se emplearon dos desinfectantes con compuestos de amonio cuaternario, siendo Agri'germ 1510 el más eficiente. Probablemente, la complejidad de su formulación y concentración de cada uno de sus ingredientes activos (glutaraldehído 15 $\%$, cloruro de alquil dimetil bencil amonio $8 \%$, cloruro de dimetil didecil amonio $2 \%$ ) resultó en una mayor efectividad contra macroconidios y clamidosporas de las cepas de Fusarium spp., utilizadas en esta investigación, en función de los parámetros evaluados. Éste fenómeno podría explicarse, en primer lugar, por el efecto del glutaraldehído que radica en la destrucción de los 
Tabla 4.- Regresión logística - probabilidad y valores de la ecuación de regresión logística

\begin{tabular}{|c|c|c|c|c|c|}
\hline \multicolumn{6}{|c|}{ Tabla de clasificación } \\
\hline \multirow[b]{2}{*}{ SCIC Observado } & \multicolumn{2}{|c|}{ SCIC Pronosticado } & \multirow{2}{*}{$\begin{array}{c}\text { Corrección de } \\
\text { porcentaje }\end{array}$} & & \\
\hline & $\begin{array}{c}\text { Con } \\
\text { crecimiento }\end{array}$ & $\begin{array}{c}\text { Sin } \\
\text { crecimiento }\end{array}$ & & & \\
\hline Con crecimiento & 582 & 49 & 92,2 & & \\
\hline Sin crecimiento & 58 & 211 & 78,4 & & \\
\hline \multirow[t]{2}{*}{ Porcentaje global } & & & 88,1 & & \\
\hline & $\begin{array}{c}\text { Valores del } \\
\text { coeficiente B }\end{array}$ & Error estándar & $\begin{array}{l}\text { Grados de } \\
\text { libertad (gl) }\end{array}$ & Sig. & $\begin{array}{c}\text { Ecuación de la } \\
\text { regresión logística } \\
(\text { Exp-B) }\end{array}$ \\
\hline Dilución & $-1,38$ & 0,11 & 1 & 0 & 0,25 \\
\hline Tiempo exposición & 0,98 & 0,16 & 1 & 0 & 2,65 \\
\hline Desinfectante & $-2,14$ & 0,16 & 1 & 0 & 0,12 \\
\hline Constante & 6,9 & 0,65 & 1 & 0 & 994,22 \\
\hline
\end{tabular}

componentes celulares, alterando la síntesis proteica de bacterias, micobacterias, virus y hongos (Cowan et al. 1993; Alvares 2018). En conexión con esto, se ha observado que glutaraldehído en una concentración de 0,25\% tiene un efecto esporicida del $100 \%$ frente a F. oxysporum f. sp. narcissi (Linfield 1991). Adicionalmente, los compuestos de amonio cuaternario, en este caso cloruro de alquil dimetil bencil amonio y cloruro de dimetil didecil amonio, al ser tensoactivos reducen la tensión superficial, lo que facilita la adsorción a las superficies y causan la ruptura de las membranas celulares de los microorganismos expuestos (Nogueira 2013; Zubel 2020). En sinergia, los compuestos de amonio cuaternario y los del grupo de los aldehídos han demostrado ser idóneos al disminuir los niveles de contaminación microbiana en superficies y utensilios dentro de laboratorios clínicos (Finch y Fay 1994; Takahashi y Fava 2011; Christen et al. 2017). Igualmente, el efecto de la combinación de estos compuestos químicos es capaz de reducir el desarrollo de resistencia, en aquellos microorganismos que pueden generar mecanismos para contrarrestar el efecto desinfectante de los aldehídos y en aquellos que puedan formar biofilms en superficies u otros objetos (Kilvington et al. 2013; Ocampo et al. 2019). Estos mecanismos de resistencia adquirida pueden ser el resultado de mutaciones de genes cromosómicos o por la adquisición de material genético en la forma de transposones (Vijayakumar y Sandle 2018).

Estudios previos realizados en Australia determinaron que los productos compuestos a base de amonio cuaternario (Banana Basher Cleaner, Banana Shed/Equipment Cleaner, Organic Food Fungal Cleaner, Sporekill, entre otros) son significativamente más efectivos inhibiendo el crecimiento de propágulos de clamidosporas de Foc R1 y Foc R4T, en condiciones similares a nuestro estudio, en todos los tiempos de exposición evaluados (30 segundos, 5 minutos, 30 minutos y 24 horas) y todas las diluciones empleadas (1:10, 1:100 y con menor impacto en 1:1000) (Nel et al. 2007; Meldrum et al. 2013; Nguyen et al. 2019). Los resultados en cuanto al segundo producto de Amonio cuaternario (cloruro de alquil dimetil bencil amonio $20 \%$ ) evaluado en este estudio son consistentes con ensayos anteriores, debido a que se evidencia la efectividad del producto al emplear diluciones $\geq$ 1:1000 o 0,1 \% dependiendo de la cepa. Igualmente, esto sugiere que la concentración de ingrediente activo es una variable a tomar en cuenta. En relación con eso, previamente se ha observado que aquellos productos, con concentraciones $\geq$ $10 \%$ de amonio cuaternario (cloruro de benzalconio, cloruro de didecil dimetil amonio, cloruro de alquil bencil dimetil amonio), con un tiempo de exposición comprendido entre 30 segundos y 24 horas presentan mayor efectividad frente a Foc (Nguyen et al. 2019). Además, es importante destacar que los resultados con este desinfectante variaron dependiendo de la cepa. Por ejemplo, la cepa B-MB-01 presentó una CMI general de 0,2 \% y en el resto de las cepas se evidenció una CMI que osciló entre 0,15 \% y 0,1 \% en macroconidios y clamidosporas (Tabla 3). Esto sugiere que la concentración a ser aplicada debería ser aquella que demuestre eficiencia frente al más amplio espectro de cepas de Fusarium spp. Además, se pudo determinar que la CMI de todos 
los desinfectantes, sobre las dos estructuras fúngicas evaluadas varió en función del tiempo de exposición (principalmente a los 30 segundos), resultando en una mayor resistencia por parte de las clamidosporas en función de la estructura de su pared y la presencia de biomoléculas de reserva (Warman y Aitken 2018).

En lo referente a Neuthox, este producto resultó ser el segundo desinfectante más eficiente en los 30 segundos de exposición en las concentraciones C2 (45 \%) frente a macroconidios de las cepas B-MB-01, FP 14-062 y YC3, y C3 (30 \%) en los aislamientos restantes (Tabla 3). Mientras que, frente a clamidosporas fueron C1 (60 \%) en las cepas FP 15-0317 y FP 14-062, y C2 (45 $\%)$ en el resto de cepas (Tabla 3). Sin embargo, presenta mayor efectividad en todas las cepas dentro de la dilución recomendada (30 \% - C3) a partir del minuto de exposición frente a macroconidios y desde los 5 minutos de exposición frente a clamidosporas. Este desinfectante está compuesto por ácido hipocloroso $(\mathrm{HClO})$, el cual forma parte de un nuevo conjunto de sustancias microbicidas que por su amplio espectro, rápida acción, amplio margen de seguridad, concentración y forma de estabilización, puede ser utilizado en procesos de desinfección y/o para controlar y prevenir un amplio número de infecciones en piel y mucosas (Calderón 2010). Los resultados obtenidos en el presente trabajo experimental con el producto Neuthox son consistentes con los hallazgos concluyentes de ensayos previos. En ese contexto, ciertos estudios demuestran que el $\mathrm{HClO}$ es capaz de reducir en $\geq 99,99 \%$ el número de macroconidios viables de Fusarium solani, en condiciones de laboratorio, a una concentración de 0,01% a partir del minuto de exposición y a concentraciones de 0,004 a 0,006 \% a partir de los 10 minutos de exposición (Robson et al. 2007; Audenaert et al. 2012; Odorcic et al. 2015; Guerra et al. 2019). Las concentraciones de ácido hipocloroso que inhibieron el desarrollo de Fusarium spp. en el presente estudio son: 0,03\%; 0,023\%; 0,015\% y 0,008 \%. De forma general, los compuestos de cloro son poderosos germicidas de amplio espectro de actividad, reactivos con la materia orgánica y deben usarse sobre superficies limpias o en concentraciones elevadas para garantizar un proceso de desinfección eficiente (Torres y Araujo 2008). El cloro y sus derivados ejercen su efecto inhibiendo reacciones enzimáticas, desnaturaliza proteínas e inactiva ácidos nucleicos (Medina y Valencia 2008; Flamenco y Guevara 2011)

En esta investigación, los productos a base de dióxido de cloro (Clodos Puro y Biocidox) no inhibieron el crecimiento de ninguna de las cepas de Fusarium spp. en ninguna de las concentraciones y tiempos de exposición evaluados. Sin embargo, otros estudios han determinado que las concentraciones óptimas de dióxido de cloro, diluido en agua destilada, son: 1 ppm (0,0001\%), 3 ppm (0,0003 \%) y 5 ppm (0,0005 \%) durante 4 minutos frente a macroconidios, micelio y clamidosporas de Fusarium spp. respectivamente (Copes et al. 2004; Scarlett et al. 2016). Asimismo, cabe añadir que ensayos in vitro llevados a cabo con dióxido de cloro gaseoso demostraron que la aplicación de este compuesto es capaz de inhibir significativamente, en concentraciones superiores a 0,0001\%, el crecimiento de Fusarium oxysporum f. sp. batatas, durante un tiempo de exposición de 30 minutos (Lee et al. 2019). Mientras que, en este estudio se trabajó con Clodos Puro con un rango de 0,0000028 (C6 producto diluido al 0,038 \%) a 0,000044 \% (C1 - producto diluido al 0,58 \%) de dióxido de cloro, concentraciones considerablemente inferiores con relación a estudios previos. Además, no se observó inhibición del crecimiento de macroconidios y clamidosporas de ninguna de las cepas de Fusarium spp. con el producto Biocidox, en concentraciones de 1 ppm, 1,5 ppm y 2 ppm de dióxido de cloro, mostrando diferencias con el estudio publicado por Scarlett et al. (2016). Adicionalmente, en el estudio llevado a cabo con dióxido de cloro gaseoso (Lee et al. 2019), la concentración y el tiempo de exposición para impedir el desarrollo del hongo son más altos que los evaluados en el presente estudio en el que se utilizó dióxido de cloro en solución. En cuanto a Clodos Puro y Biocidox es necesario evaluar concentraciones superiores a 2 ppm (0,0002 \%) durante los mismos tiempos de exposición, con el fin de determinar la concentración mínima inhibitoria. Estudios realizados en Australia demostraron que un aumento en el tiempo de contacto a 24 horas mejoró la eficacia de la mayoría de los desinfectantes contra Foc R1 y R4T en comparación con los tiempos de contacto más cortos (Nguyen et al. 2019).

Nuestros resultados fueron respaldados mediante el uso de controles de fenol (control positivo: dilución 1:60 y control negativo: dilución 1:70), compuesto que se emplea como desinfectante a 
altas concentraciones debido a su capacidad de desnaturalizar proteínas y romper las membranas celulares (Kahrs 2008; Robles et al. 2018). Además, a partir de los tubos seleccionados entre los controles positivos y aquellos que presentaban crecimiento fúngico, se comprobó la presencia únicamente de Fusarium spp. mediante observación macroscópica y microscópica, luego de ser sembrados en superficie en agar PDA, basándose en la metodología ISO 21527-2 (2018).

Es necesario realizar estudios posteriores tomando en cuenta condiciones ambientales más realistas. Por ejemplo la materia orgánica puede interferir en la efectividad del desinfectante al neutralizarlo (Bennett et al. 2011; Meldrum et al. 2013; Nguyen et al. 2019), principalmente por su efecto quelante (Tezel y Pavlostathis 2015). Adicionalmente, el tiempo de contacto junto a otros factores, como la temperatura del agua, el pH y el contenido mineral, afectan las propiedades germicidas de los desinfectantes, (Copes et al. 2004; Berrang et al. 2011; Scarlett et al. 2016). Estos factores no fueron considerados en esta investigación, pero podrían afectar la actividad del desinfectante. Además de la eficacia del producto, se debe considerar su coste, la corrosividad, la longevidad y el impacto ambiental.

\section{Conclusión}

Este ensayo ha evaluado la eficacia de varios desinfectantes comerciales frente a macroconidios y clamidosporas de cinco cepas de Fusarium spp. Se determinó que Agri'germ 1510, compuesto por glutaraldehído $15 \%$ y dos tipos de amonio cuaternario (cloruro de alquil dimetil bencil amonio $8 \%$ y cloruro de dimetil didecil amonio $2 \%$ ), fue el desinfectante más efectivo inhibiendo a todas las cepas de Fusarium spp. en todos los tiempos de exposición con la concentración recomendada y las diluciones menos concentradas. Neuthox (ácido hipocloroso 0,05 \%) fue el segundo desinfectante más eficiente, mejorando su efecto a partir del minuto de exposición dentro de las concentraciones evaluadas. El producto Amonio cuaternario (cloruro de alquil dimetil bencil amonio $20 \%$ ) exhibe resultados más eficientes en las concentraciones superiores y recomendada a partir de los 5 minutos. De forma general, las clamidosporas de Fusarium spp. presentaron mayor resistencia al contacto con desinfectantes durante los 30 segundos de contacto, a diferencia de macroconidios. Asimismo, se comprobó que el efecto desinfectante de los productos mejora a medida que pasa el tiempo de exposición, y disminuye al diluirse. No obstante, se pueden tomar medidas preventivas confiables en base a los resultados de este estudio, debido a la congruencia que presentan con estudios anteriores ensayados contra cepas de Foc R4T, con el fin de evitar la entrada de este patógeno al territorio ecuatoriano.

\section{Agradecimientos}

Agradecemos a los directivos de la Agencia de Regulación y Control Fito y Zoosanitario del Ecuador por permitir la realización de este ensayo dentro de sus instalaciones y gestionar la adquisición de los distintos productos desinfectantes evaluados.

\section{Declaración de conflicto de intereses}

Los autores declaran no tener conflictos de interés entre la realización de este artículo y su financiamiento o afiliaciones laborales.

\section{Contribución de los autores}

Martín Marcial-Coba: Concepción y diseño del estudio, Diseño experimental del estudio, Análisis e interpretación de datos, Redacción de la primera versión del manuscrito, Revisión del manuscrito.

Alexander Toaza: Concepción y diseño del estudio, Diseño experimental del estudio, Análisis e interpretación de datos, Redacción de la primera versión del manuscrito, Revisión del manuscrito, Gestión del financiamiento.

Galo Ordoñez: Adquisición / Colección de datos, Análisis e interpretación de datos, Redacción de la primera versión del manuscrito.

Jeniffer Yánez: Revisión del manuscrito, Gestión del financiamiento.

Elena Granda: Revisión del manuscrito.

\section{Referencias bibliográficas}

AOAC. 2005. Association of Official Analytical Chemist, Official Methods of Analysis. 18th Edition, 
AOAC International, Suite 500, 481 North Frederick Avenue, Gaithersburg, Maryland 20877-2417, USA.

Al-daour A, Al-shawwa M, Abu-Naser S. 2019. Banana Classification Using Deep Learning. $3(12): 1-8$

Alvarez H. 2018. Efectividad de los desinfectantes alternativos:ácido peracético u ortoftalaldehído en comparación con el glutaraldehído para lograr una desinfección óptima y sin daños, en el endoscopios flexibles. Tesis de pregrado. Facultad de Ciencias de la Salud. Universidad Norbert Wiener. Lima, Perú.

Amani M, Avagyan G. 2014. Isolation and Identification of Fungal Pathogens on Banana Trees (Musa acuminata L.) in Iran. Int Acad Journals Int J AgriScience. 4(8):409-413.

Angel M, Rodríguez D. 2016. Aviso público del riesgo y situación actual. Servicio nacional de sanidad, inocuidad y calidad agroalimentaria. México.

Audenaert K, Monbaliu S, Deschuyffeleer N, Maene P, Vekeman F, Haesaert G, De Saeger S, Eeckhout M. 2012. Neutralized electrolyzed water efficiently reduces Fusarium spp. in vitro and on wheat kernels but can trigger deoxynivalenol (DON) biosynthesis. Food Control. 23(2):515521. doi:10.1016/j.foodcont.2011.08.024.

Bennett RS, O'Neill W, Smith L, Hutmacher RB. 2011. Plant pathology and nematology: Activity of commercial detergents against conidia and chlamydospores of Fusarium oxysporum f. sp. vasinfectum. J Cotton Sci. 15(2):162-169.

Berrang ME, Meinersmann RJ, Cox NA, Fedorka-Cray PJ. 2011. Application of chlorine dioxide to lessen bacterial contamination during broiler defeathering. J Appl Poult Res. 20(1):33-39. doi:10.3382/japr.2010-00178.

Burguet Lago N, Brito Godoy LC, Cánovas Borges I. 2013. Evaluation of the effectiveness of a disinfectant through the contact plate method. Rev Cuba Farm. 47(2):185-192.

Calderón JL. 2010. Artículo de revisión ÁCIDO HIPOCLOROSO (HOCI) "Una nueva alternativa en antisepsia y desinfección desarrollada en Colombia."Lab Actual. (42):27-31. doi:10.18004/rvspmi/

Cedeño A. 2020. Medidas de prevención y contingencia de Fusarium oxysporum raza 4 en el cultivo de Banano, en la hacienda Elbas. Tesis de pregrado. Facultad de Ciencias Agropecuarias. Universidad Técnica de Babahoyo. Ecuador.

Christen V, Faltermann S, Brun NR, Kunz PY, Fent K. 2017. Cytotoxicity and molecular effects of biocidal disinfectants (quaternary ammonia, glutaraldehyde, poly (hexamethylene biguanide) hydrochloride PHMB) and their mixtures in vitro and in zebrafish eleuthero-embryos. Sci Total Environ. 586:1204-1218. doi:10.1016/j.scitotenv.2017.02.114.

Copes WE, Chastaganer GA, Hummel RL. 2004. Activity of chlorine dioxide in a solution of ions and pH against Thielaviopsis basicola and Fusarium oxysporum. Plant Dis. 88(2):188-194. doi:10.1094/PDIS.2004.88.2.188.

Cowan RE, Manning AP, Ayliffe GAJ, Axon ATR, Causton JS, Cripps NF, Hall R, Hanson PJV, Harrison J, Leicester RJ, et al. 1993. Aldehyde disinfectants and health in endoscopy units the report of a working party of the british society of gastroenterology endoscopy committee. Gut. 34(11):16411645. doi:10.1136/gut.34.11.1641.

Davicino R, Mattar MA, Casali YA, Correa SG, Pettenati EM, Micalizzi B. 2007. Actividad antifúngica de extractos de plantas usadas en medicina popular en Argentina. Rev Peru Biol. 14(2):247-251. doi:10.15381/rpb.v14i2.1784. 
Dita, M. A.; Ramos, E. E.; Pérez LF. 2013. Plan de contingencia ante un brote de la raza 4 tropical de Fusarium oxysporum f. sp. cubense. Organismo internacional regional de sanidad agropecuaria, OIRSA. San Salvador, El Salvador.

Dita M, Barquero M, Heck D, Mizubuti ESG, Staver CP. 2018. Fusarium wilt of banana: Current knowledge on epidemiology and research needs toward sustainable disease management. Front Plant Sci. 871(October):1-21. doi:10.3389/fpls.2018.01468.

Dita M, Martinez De La Parte E. 2014. Technical Manual Prevention and diagnostic of Fusarium Wilt (Panama Disease) of banana caused by Fusarium oxysporum f. sp. cubense Tropical Race 4(TR4). FAO. 4(May):1-74.

Dita MA, Waalwijk C, Buddenhagen IW, Souza JT, Kema GHJ. 2010. A molecular diagnostic for tropical race 4 of the banana fusarium wilt pathogen. Plant Pathol. 59(2):348-357. doi:10.1111/ j.1365-3059.2009.02221.x.

FAO. 2019. Análisis del mercado del banano: resultados preliminares 2019. Organización de las Naciones Unidas para la Alimentación y la Agricultura. Roma.

Fernandez-Crehuet M, Espigares M, Moreno E, Espigares E. 2013. Specificity of the neutralizers as the cause of errors in evaluating disinfectant efficacy: an assessment of triclosan. Lett Appl Microbiol. 57:517-525.

Finch W, Fay Z. 1994. United States Patent: Fortified glutaraldehyde chemical sterlant/disinfectant. (19).

Flamenco J, Guevara G. 2011. Formulación de Tres Productos Desinfectantes y Evaluación de Su Actividad Antimicrobiana. Tesis de pregrado. Facultad de Química y Farmacia. Universidad de El Salvador. El Salvador.

Gonsabay R. 2014. Cultivo del banano en Ecuador. AFESE::113-142.

Guentzel JL, Lam KL, Callan MA, Emmons SA, Dunham VL. 2010. Postharvest management of gray mold and brown rot on surfaces of peaches and grapes using electrolyzed oxidizing water. Int J Food Microbiol. 143(1-2):54-60. doi:10.1016/j.ijfoodmicro.2010.07.028.

Guerra NR, Chávez M, Treviño RS, Garcia JF, Torres JA, Méndez A, Marroquín AG. 2019. Effects of Neutral Electrolysed Water on tomato seeds artificially contaminated with Fusarium and Aspergillus. Seed Sci Technol.:211-227. doi:10.15258/sst.2019.47.2.08

Hwang SC, Ko WH. 2004. Cavendish banana cultivars resistant to fusarium wilt acquired through somaclonal variation in Taiwan. Plant Dis. 88(6):580-588. doi:10.1094/PDIS.2004.88.6.580.

IBM Corp. Released 2015. IBM SPSS Statistics for Windows, Version 23.0. Armonk, NY: IBM Corp.

ICA. 2019. La Federación Nacional de Cafeteros se une a la campaña para la prevención del Fusarium R4T. Inst Colomb Agropecu. Colombia.

ICA. 2020. Resolución No.072820. https://www.ica.gov.co/getattachment/be3be9ed-b1aa-43f199f6-637bfde76318/2020R72820.aspx

ISO 21527-2. 2008. Microbiology of food and animal feeding stuffs - Horizontal method for the enumeration of yeast and molds. Part 1: Colony count technique in products with water activity greater than 0,95.

Kahrs R. 2008. Principios generales de la desinfección. Organ Mund Sanid Anim. 14(1):143-163. 
Kilvington S, Lam A, Nikolic M, Brady N. 2013. Resistance and growth of Fusarium species in contact lens disinfectant solutions. Optom Vis Sci. 90(5):430-438. doi:10.1097/OPX.0b013e31828f4dfe.

Lee YJ, Jeong JJ, Jin H, Kim W, Jeun YC, Yu GD, Kim KD. 2019. In vitro and in vivo inhibitory effects of gaseous chlorine dioxide against Fusarium oxysporum f. Sp. batatas isolated from stored sweetpotato: Study II. Plant Pathol J. 35(5):437-444. doi:10.5423/PPJ.OA.04.2019.0078.

Li R, Baysal-Gurel F, Abdo Z, Miller SA, Ling KS. 2015. Evaluation of disinfectants to prevent mechanical transmission of viruses and a viroid in greenhouse tomato production. Virol J. 12(1). doi:10.1186/s12985-014-0237-5.

Lin Y, Hussain M, Avery PB, Qasim M, Fang D, Wang L. 2016. Volatiles from plants induced by multiple aphid attacks promote conidial performance of Lecanicillium lecanii. PLoS One. $11(3): 1-$ 14. doi:10.1371/journal.pone.0151844.

Linfield C. 1991. A Comparative Study of the Effects of Five Chemicals on the Survival of Chlamydospores of Fusarium oxysporum f. sp. narcissi. J Phytopathol. 131:297-304.

Loor, E.; Gordon T. 2013. Evaluación del proceso de limpieza y desinfección en el área estéril del laboratorio farmacéutico Maquipharma S.A.

Medina L, Valencia L. 2008. Evaluación de la eficacia de un desinfectante de alto nivel, a base de peróxido de hidrógeno, empleado en la esterilización de dispositivos e instrumentos hospitalarios.

Meldrum RA, Daly AM, Tran-Nguyen LTT, Aitken EAB. 2013. The effect of surface sterilants on spore germination of Fusarium oxysporum f. sp. cubense tropical race 4. Crop Prot. 54:194-198. doi:10.1016/j.cropro.2013.08.014.

Miranda B. 2019. Fusarium R4T, el devastador hongo por el que declaran la primera emergencia nacional en América Latina y que preocupa a la industria del banano - BBC News Mundo. BBC News Mundo.

Molina, B.; Sinohin, V:; Baroña L. 2009. Status of Tropical race 4 of Panama Wilt in Asia. Prevention. (June):1.

Moore NY, Pegg KG, Smith LJ, Langdon PW, Bentley S, Smith MK. 2001. Fusarium wilt of banana in Australia.

Nel B, Steinberg C, Labuschagne N, Viljoen A. 2007. Evaluation of fungicides and sterilants for potential application in the management of Fusarium wilt of banana. Crop Prot. 26(4):697-705. doi:10.1016/j.cropro.2006.06.008.

Nguyen TV., Tran-Nguyen LTT, Wright CL, Trevorrow P, Grice K. 2019. Evaluation of the Efficacy of Commercial Disinfectants Against Fusarium oxysporum f. sp. cubense Race 1 and Tropical Race 4 Propagules. Plant Dis. Vol. 103:721-728.

Nogueira D. 2013. Estudio de tensioactivos aniónicos y catiónicos derivados de lisina: interacción con membranas celulares, desarrollo de sistemas nanoestructurados para la liberación intracelular y evaluación toxicológica in vitro.

Ocampo L, Banegas M, Sumano H, Alfonseca-Silva E, Campillo-Navarro M, Soria-Castro R, Aquino I. 2019. Evaluation of mycobactericidal and brucellicidal efficacy of an aldehyde and quaternary ammonium solution and a mixture of phenolic compounds. Isr J Vet Med. 74(4):190-195.

Odorcic S, Haas W, Gilmore MS, Dohlman CH. 2015. Fungal infections after boston type 1 keratoprosthesis implantation: Literature review and in vitro antifungal activity of hypochlorous 
acid. Cornea. 34(12):1599-1605. doi:10.1097/ICO.0000000000000639.

ONU. 2019. Fácil de propagar y difícil de eliminar, la nueva plaga que amenaza el banano y el plátano en América Latina. Not ONU.

Pérez L. 2015. Fusarium Wilt (Panama Disease) of Bananas: An Updating Review of the Current Knowledge on the Disease and Its Causal Agent.

Pérez L. 2015. Las Mejores Prácticas Para la Prevención de la Raza 4 Tropical de la Marchitez por Fusarium y otras enfermedades exóticas en fincas bananeras. Fitosanidad. 19: 243-250

Ploetz RC. 2006. Fusarium wilt of banana is caused by several pathogens referred to as Fusarium oxysporum f. sp. cubense. Phytopathology. 96(6):653-656. doi:10.1094/PHYTO-96-0653.

Robles MDM, Villegas ES, Cortez OM, Castellanos IEG, Plascencia PCM, Esparza LMA. 2018. Evaluación de la eficiencia de desinfectantes comerciales para uso alimentario en el control de crecimiento Escherichia coli. Av Investig en Inocuidad Aliment. 1(1):5-8.

Robson MC, Payne WG, Ko F, Mentis M, Donati G, Shafii SM, Culverhouse S, Wang L, Khosrovi B, Najafi R, et al. 2007. Hypochlorous Acid as a Potential Wound Care Agent: Part II. Stabilized Hypochlorous Acid: Its Role in Decreasing Tissue Bacterial Bioburden and Overcoming the Inhibition of Infection on Wound Healing. J Burns Wounds. 6:e6.

Rodriguez MAD, Vicente LP, Martínez E. 2014. INOCULATION OF Fusarium oxysporum f. sp. cubense CAUSAL AGENT OF FUSARIUM WILT IN BANANA. Worshop Diagnosis Fusarium Wilt. 4(May):1-74.

Rood L, Koutoulis A, Bowman JP, Evans DE, Stanley RA, Kaur M. 2018. Control of microbes on barley grains using peroxyacetic acid and electrolysed water as antimicrobial agents. Food Microbiol. 76:103-109. doi:10.1016/j.fm.2018.05.002.

Scarlett K, Collins D, Tesoriero L, Jewell L, van Ogtrop F, Daniel R. 2016. Efficacy of chlorine, chlorine dioxide and ultraviolet radiation as disinfectants against plant pathogens in irrigation water. Eur J Plant Pathol. 145(1):27-38. doi:10.1007/s10658-015-0811-8.

Rodriguez M. 2012. Mal De Panamá: Medidas De Control Y Prevención Información. Servicio Técnico de Agricultura y Desarrollo Rural. Cabildo de Tenerife. España.

Soto M. 2011. Situación y Avances Tecnologicos en la Producción Bananera Mundial. Rev Bras Frutic. 33(SPEC. ISSUE 1):013-028. doi:10.1590/s0100-29452011000500004.

Takahashi D, Fava C. 2011. Uso de glutaraldehído en la desinfección de incubadoras. XXII Congreso Latinoamericano de Avicultura 2011. Buenos Aires, Argentina.

Tezel U, Pavlostathis S. 2015. Quaternary ammonium disinfectants: microbial adaptation, degradation and ecology. Curr Opin Biotechnol. 33:296-304.

Torres N, Araujo F. 2008. Evaluación de los desinfectantes utilizados en el proceso de limpieza y desinfección del área de fitoterapéuticos en laboratorios Pronabell. LTAD.

Vásquez A, Villarreal T, Rodríguez G. 2016. Effectiveness of neutral electrolyzed water on incidence of fungal rot on tomato fruits (Solanum lycopersicum L.). J Food Prot. 79(10):1802-1806. doi:10.4315/0362028X.JFP-15-494.

Vásquez R. 2010. El impacto del comercio del Banano en el desarrollo del Ecuador. AFESE Temas Int. 53(53):167-182.

Vijayakumar R, Sandle T. 2018. A review on biocide reduced susceptibility due to plasmidborne 
antiseptic-resistant genes_-special notes on pharmaceutical environmental isolates. J Appl Microbiol. 126:1011-1022.

Villaverde J. 2018. Fusarium. Todo lo que necesitas saber sobre este género. Plantamus. https:// plantamus.com/blog/todo-sobre-fusarium/

Warman N, Aitken E. 2018. The Movement of Fusarium oxysporum f.sp. cubense (Sub-Tropical Race 4) in Susceptible Cultivars of Banana. Front Plant Sci. 9(1748):1-9.

Zubel I. 2020. Effect of cations on silicon anisotropic etching process in solutions containing TMAH and TMAH with tensioactive compounds. Sensors Actuators, A Phys. 303:111829. doi:10.1016/j. sna.2020.111829. 